\title{
(6) OPEN ACCESS \\ The effects of tumour necrosis factor inhibitors, methotrexate, non-steroidal anti-inflammatory drugs and corticosteroids on cardiovascular events in rheumatoid arthritis, psoriasis and psoriatic arthritis: a systematic review and meta-analysis
}

\author{
Camille Roubille, ${ }^{1}$ Vincent Richer, ${ }^{2}$ Tara Starnino, ${ }^{3}$ Collette McCourt, ${ }^{4}$ \\ Alexandra McFarlane, ${ }^{5}$ Patrick Fleming, ${ }^{6}$ Stephanie Siu, ${ }^{7}$ John Kraft, ${ }^{8}$ Charles Lynde, ${ }^{8}$ \\ Janet Pope, ${ }^{7}$ Wayne Gulliver, ${ }^{9}$ Stephanie Keeling, ${ }^{5}$ Jan Dutz, ${ }^{4}$ Louis Bessette, ${ }_{1}^{10}$ \\ Robert Bissonnette, ${ }^{11}$ Boulos Haraoui ${ }^{12}$
}

\begin{abstract}
Handling editor Tore K Kvien
- Additional material is published online only. To view please visit the journal online (http://dx.doi.org/10.1136/ annrheumdis-2014-206624).

For numbered affiliations see end of article.
\end{abstract}

\section{Correspondence to} Boulos Haraoui, Institut de Rhumatologie de Montreal, 1551 Ontario Street East, Montreal, Quebec, Canada H2L 1S6;

boulos.haraoui@ssss.gouv.qc.ca

Received 15 September 2014 Revised 15 December 2014 Accepted 16 December 2014 Published Online First

5 January 2015

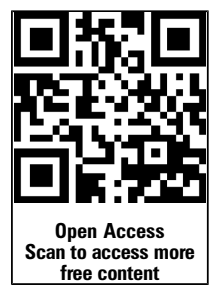

CrossMark

To cite: Roubille $C_{\text {, }}$ Richer V, Starnino T, et al. Ann Rheum Dis

2015;74:480-489.

\section{ABSTRACT}

The objective of this systematic literature review was to determine the association between cardiovascular events (CVEs) and antirheumatic drugs in rheumatoid arthritis

(RA) and psoriatic arthritis (PsA)/psoriasis (Pso).

Systematic searches were performed of MEDLINE, EMBASE and Cochrane databases (1960 to December 2012) and proceedings from major relevant congresses (2010-2012) for controlled studies and randomised trials reporting confirmed CVEs in patients with RA or PsA/Pso treated with antirheumatic drugs. Randomeffects meta-analyses were performed on extracted data.

Out of 2630 references screened, 34 studies were included: 28 in RA and 6 in PsA/Pso. In RA, a reduced risk of all CVEs was reported with tumour necrosis factor inhibitors (relative risk (RR), $0.70 ; 95 \% \mathrm{Cl} 0.54$ to 0.90 ; $\mathrm{p}=0.005)$ and methotrexate (RR, $0.72 ; 95 \% \mathrm{Cl} 0.57$ to $0.91 ; p=0.007)$. Non-steroidal anti-inflammatory drugs (NSAIDs) increased the risk of all CVEs (RR, 1.18; 95\% Cl 1.01 to $1.38 ; p=0.04$ ), which may have been specifically related to the effects of rofecoxib. Corticosteroids increased the risk of all CVEs (RR, 1.47; 95\% Cl 1.34 to 1.60; $p<0.001)$. In PsA/Pso, systemic therapy decreased the risk of all CVEs (RR, 0.75; 95\% Cl 0.63 to 0.91; $p=0.003)$.

In RA, tumour necrosis factor inhibitors and methotrexate are associated with a decreased risk of all CVEs while corticosteroids and NSAIDs are associated with an increased risk. Targeting inflammation with tumour necrosis factor inhibitors or methotrexate may have positive cardiovascular effects in RA. In PsA/Pso, limited evidence suggests that systemic therapies are associated with a decrease in all CVE risk.

\section{INTRODUCTION}

Patients with rheumatoid arthritis (RA) have increased risk of cardiovascular morbidity and mortality. ${ }^{1}{ }^{2}$ Although less evidence has been published so $\mathrm{far}^{3}{ }^{4}$ this increased risk is also suspected in patients with psoriasis (Pso), with or without psoriatic arthritis (PsA). Irrespective of classical cardiovascular risk factors, the systemic inflammation characteristic of RA and Pso/PsA plays a pivotal role in increasing cardiovascular risk by accelerating atherosclerosis. ${ }^{5}$ Vascular inflammation and the related elevated cardiovascular risk may affect all patients with RA, beginning in the early stage of disease (perhaps even preceding clinical onset) ${ }^{6}$ and worsening with additional classical cardiovascular risk factors.

Many anti-inflammatory strategies have emerged as potential therapeutic approaches for atherosclerosis. ${ }^{7}$ Likewise, treatment of the underlying inflammatory process could contribute to improved cardiovascular outcomes in patients with RA and Pso/PsA. ${ }^{8}$ This is reflected in one of the current European League Against Rheumatism recommendations in RA, ${ }^{9} 10$ which advises achieving remission or low disease activity as early as possible, not only for better structural and functional outcomes, but also to reduce cardiovascular risk. However, it is still open to discussion as to whether targeting systemic inflammation itself with disease-modifying antirheumatic drugs (DMARDs) reduces the occurrence of cardiovascular events (CVEs) in patients with RA or Pso/PsA.

The purpose of this systematic literature review and meta-analysis was to explore the association between the use of biologics (including tumour necrosis factor (TNF) inhibitors), non-biological DMARDs (including methotrexate), corticosteroids and non-steroidal anti-inflammatory drugs (NSAIDs), and CVEs in patients with RA or Pso/PsA.

\section{METHODS}

A systematic literature review and meta-analysis were performed according to Preferred Reporting Items for Systematic reviews and Meta-Analyses statement. ${ }^{11}$

\section{Data sources and searches}

A systematic literature search of MEDLINE (via PubMed), EMBASE and the Cochrane Library databases (1960 to December 2012) was performed to identify observational studies and randomised controlled trials that reported CVEs in adults with RA or Pso/PsA treated with biologics (including TNF inhibitors), non-biological DMARDs (including methotrexate), NSAIDs and corticosteroids (see online supplementary eMethods). Searches were restricted to English language. We also searched the 
proceedings of the American College of Rheumatology, European League Against Rheumatism, American Academy of Dermatology and European Academy of Dermatology and Venereology annual meetings (2010-2012) and hand-searched reference lists for relevant additional studies.

\section{Study selection}

Studies were included if they were observational studies or randomised controlled trials that reported relevant confirmed CVEs (including all CVEs, myocardial infarction, heart failure, stroke and/or major adverse cardiac events); included patients with RA or Pso/PsA treated with biologics, non-biological DMARDs, corticosteroids or NSAIDs (or phototherapy for Pso/PsA); and included a suitable control group (another treatment, such as a TNF inhibitor compared with methotrexate, or non-use of the investigative treatment, such as use of a TNF inhibitor compared with non-use of a TNF inhibitor). Studies were excluded if they only reported data on cardiovascular risk factors (eg, diabetes mellitus), intermediate endpoints (eg, lipid levels) or surrogate markers of atherosclerosis (eg, arterial intimae media thickness); reported data on $<400$ patients; had a follow-up duration $<1$ year (to ensure that impact of the assessed treatment was most likely to be a true effect and not due to chance in a short duration of observation); included a patient population with a mean age of 80 years or older (to allow homogeneous crossstudy populations, as the majority of studies included populations with a mean age of approximately 60 years); or did not have sufficient data to convert to relative risk (RR). Two studies that specifically included veteran patients with $\mathrm{RA}^{12}{ }^{13}$ were excluded because $90 \%$ of the study population comprised men, which is not representative of the classical gender stratification in RA.

One author (CR) screened all titles and abstracts for potential inclusion. Two authors ( $\mathrm{CR}$ and $\mathrm{BH})$ then independently screened the full text of the selected studies for inclusion in the systematic review and meta-analysis according to the predetermined criteria. Disagreements were resolved by consensus.

\section{Data extraction and quality assessment}

Two investigators (CR and $\mathrm{BH}$ ) independently extracted the following data for each study using a predefined data collection form: study design; data sources; sample size; age of the subjects; underlying disease (RA or Pso/PsA); duration of follow-up; treatments under investigation; cardiovascular outcomes of interest; number of outcomes and models of adjustment (age and gender, cardiovascular risk factors, history of cardiovascular disease, RA disease activity, and use of TNF inhibitors, methotrexate, corticosteroids or NSAIDs). HRs, ORs, RRs, rate ratios or mean effect sizes for each drug and comparator, together with their associated 95\% CIs, were recorded for each endpoint. When only incident outcomes were reported, the numbers of events in the compared groups were extracted. We contacted authors for missing data when necessary. If studies reported data on several drugs and/or on several cardiovascular endpoints, each value was included in the analysis, so one reference could contribute several different data values to the final meta-analysis. When several models of adjustment were reported, the most adjusted estimates were used in the analysis. Selected studies were assessed for quality using the Newcastle-Ottawa Scale, designed to assess the quality of nonrandomised studies such as cohort and case-control studies. ${ }^{14}$ Briefly, this scale allocates points for appropriateness of participant selection (0-4 points), comparability (0-2 points) and exposure or outcome (0-3 points).

\section{Data synthesis and statistical analysis}

Extracted data were combined for meta-analysis using Review Manager (RevMan) software (Cochrane Collaboration). When only incident outcomes were reported, RRs were computed. If patient-years of follow-up were available, rate ratios were calculated and used in analysis. The resulting adjusted or unadjusted RRs, HRs and rate ratios were considered equivalent measures of effect size and entered into RevMan. ORs were transformed to RRs. ${ }^{15} 16$ These measures were generically referred to as risk ratios. For all outcomes, random-effects meta-analyses were conducted. This assumes that different studies were estimating different intervention effects and partly explains the heterogeneity between studies. Forest plots were constructed to summarise the risk ratio estimates and their 95\% CIs. These figures present measures of heterogeneity across studies (Cochrane Q statistic, noted as $\chi^{2}$ and the $\mathrm{I}^{2}$ statistic) and a test for overall effect $(\mathrm{Z})$. Funnel plots and Egger's regression test were also produced to help detect potential publication bias.

\section{Primary and secondary outcomes}

The primary outcome was the association between treatment and all CVEs. The secondary outcomes were the association between treatment and myocardial infarction, heart failure, stroke or major adverse cardiac events.

\section{RESULTS}

\section{Study selection}

We identified 2630 unique references through searching databases, conference proceedings and reference lists (figure 1). Of these, 2526 were excluded as they were not relevant to our topic or related to cardiovascular risk factors or surrogate markers of atherosclerosis. Of the 104 remaining references, 66 were selected for full-text review. Finally, 34 references met the selection criteria for meta-analysis (see online supplementary table S1): 28 in RA (total of 236525 subjects; 5410 CVEs) ${ }^{17-44}$ and 6 in Pso/PsA (220 209 subjects; 2701 CVEs). ${ }^{12} 45-49$

\section{Cardiovascular outcomes in RA: results of meta-analyses}

In RA, TNF inhibitors were significantly associated with a reduction in the risk of all CVEs (RR, 0.70; 95\% CI 0.54 to $0.90 ; p=0.005)$, as well as in myocardial infarctions, strokes and major adverse cardiac events (figure 2A). No significant effect on heart failure was observed (figure 2A). A beneficial association between methotrexate and reduction in the risk of all CVEs (RR, $0.72 ; 95 \%$ CI 0.57 to $0.91 ; \mathrm{p}=0.007$ ) and myocardial infarction was also found (figure $2 \mathrm{~B}$ ). However, in contrast with TNF inhibitors, methotrexate was not associated with a significant decrease in the risk of strokes and major adverse cardiac events, although trends towards decreasing risk of heart failure were found (figure 2B). This may be due to the low number of events included in the meta-analysis, which did not provide sufficient statistical power to observe a differential effect.

NSAIDs increased the risk of all CVEs (RR, 1.18; 95\% CI 1.01 to $1.38 ; p=0.04)$ and strokes. This effect appears mostly driven by cyclooxygenase-2 (COX-2) inhibitors (RR, 1.36; 95\% CI 1.10 to $1.67 ; \mathrm{p}=0.004)$ rather than non-selective NSAIDs (RR, $1.08 ; 95 \%$ CI 0.94 to $1.24 ; \mathrm{p}=0.28$ ) (figure $2 \mathrm{C}$ ). Of note, some studies assessing rofecoxib, which has already been withdrawn from the market, have been included. Hence, we 
Figure 1 Search and selection of studies for systematic review and meta-analysis. PsA, psoriatic arthritis; Pso, psoriasis; RA, rheumatoid arthritis.

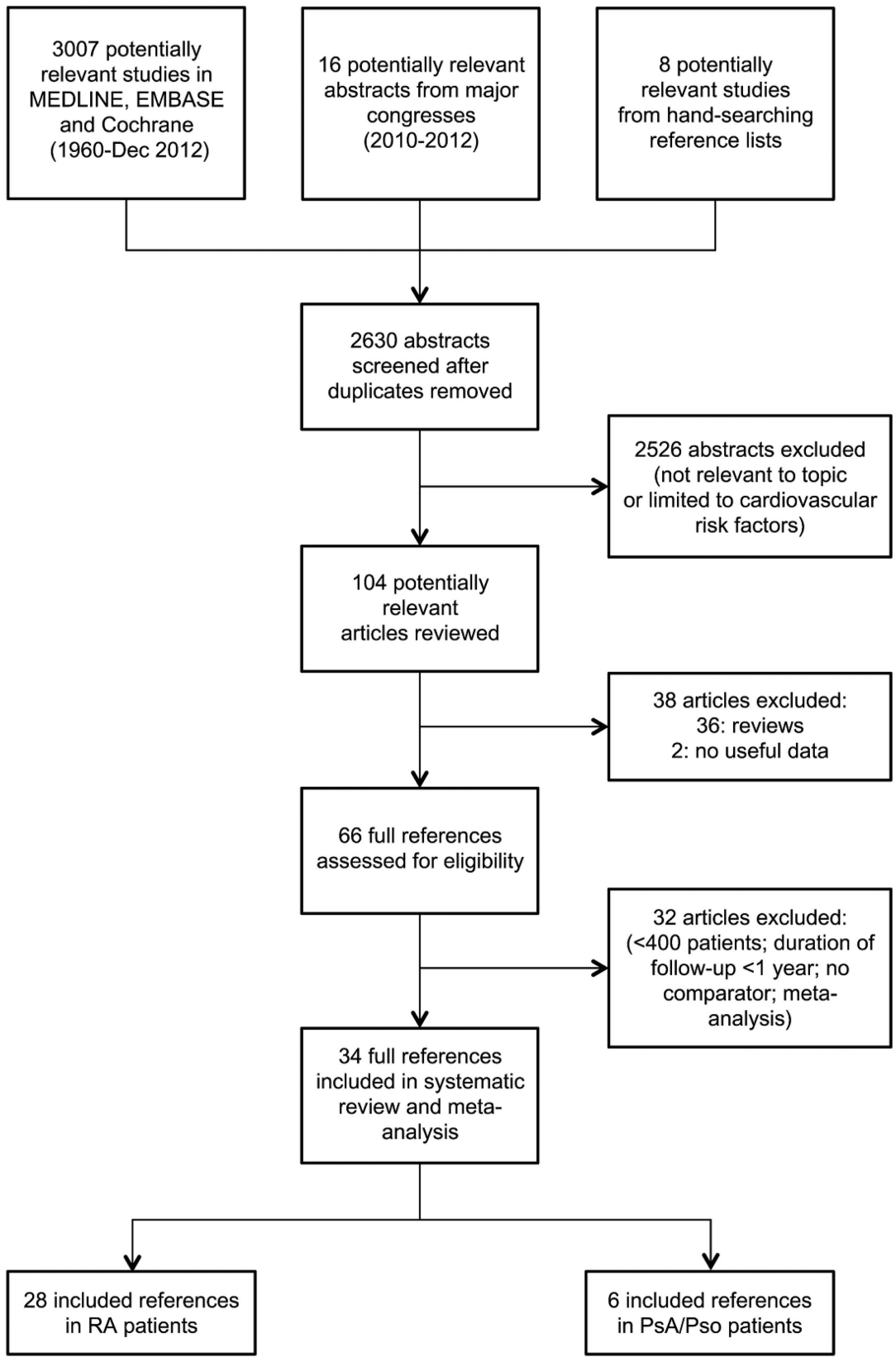

performed separate meta-analyses for rofecoxib and celecoxib. While rofecoxib increased the risk of all CVEs (RR, 1.58; 95\% CI 1.24 to $2.00 ; p<0.001)$, celecoxib did not $(R R, 1.03 ; 95 \%$ CI 0.80 to $1.32 ; \mathrm{p}=0.81$ ) (see online supplementary figure $\mathrm{S} 1$ ). Additionally, NSAIDs did not demonstrate any significant effect on risk of myocardial infarction, heart failure or major adverse cardiac events (figure 2C); however, with few studies included, these findings should be interpreted with caution.

Corticosteroids were associated with an increased risk of all CVEs (RR, 1.47; 95\% CI 1.34 to $1.60 ; \mathrm{p}<0.001$ ), as well as risk of myocardial infarction, stroke, heart failure and major adverse cardiac events (figure 2D).

In the meta-analyses evaluating TNF inhibitors or methotrexate, some heterogeneity was found between studies for all endpoints (except stroke). Heterogeneity was only observed for the all CVE endpoint in the meta-analyses evaluating all NSAIDs or COX-2 inhibitors. Of note, no significant heterogeneity was found for the all CVE endpoint in the meta-analyses evaluating non-selective NSAIDs and corticosteroids or for any specific endpoint in the meta-analyses evaluating NSAIDs and corticosteroids. Analysis for publication bias was performed using funnel plots for all CVE endpoints (figure 3). Although subjective, visual inspection did not suggest publication bias for this outcome. Egger's asymmetry coefficient, known to be low powered, did detect potential bias in the meta-analysis of corticosteroid use $(p=0.02$, figure 3$)$ and COX-2 inhibitor use $(\mathrm{p}<0.01$; see online supplementary figure $\mathrm{S} 2)$.

\section{Cardiovascular outcomes in Pso/PsA: results of meta-analyses}

Only six studies met our selection criteria in patients with Pso and/or PsA; therefore, we only had sufficient data to evaluate the effect of systemic therapy compared with no systemic therapy or topical treatment on risk of all CVEs. Systemic therapy was associated with a significant decrease in risk of all CVEs in Pso/PsA (RR, 0.75; 95\% CI 0.63 to 0.91; 
Figure 2 Meta-analyses of all cardiovascular events and individual cardiovascular events in patients with rheumatoid arthritis treated with (A) tumour necrosis factor inhibitors; (B) methotrexate; (C) non-steroidal anti-inflammatory drugs; or (D) corticosteroids in controlled studies. Size of data markers indicates relative weight of the study (from random-effects analysis). COX-2, cyclooxygenase-2; CVE, cardiovascular event; MACE, major adverse cardiac event; MTX, methotrexate; RR, relative risk; TNFi, tumour necrosis factor inhibitor.

A Tumour necrosis factor inhibitors

All CVE
Bernatsky et al. 2005
Bozaite-Gluosniene et al.
Burmester et al. 2012
Carmona et al. 2007
Dixon et al. 2007
Greenberg et al. 2011
Jacobsson et al. 2005
Listing et al. 2008
Ljung et al. $2012 b$
Low et al. 2012
Lunt et al. 2010
Nadareishvili et al. 2008
Setoguchi et al. 2008
Solomon et al. 2012
Wolfe et al. 2004
Wolfe et al. 2008
All

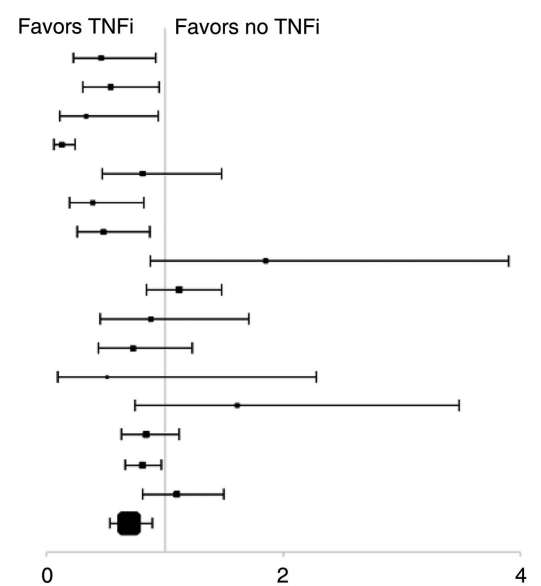

RR $[95 \% \mathrm{Cl}]$

Weight

$0.5[0.2,0.9]$

$0.54[0.30,0.95] \quad 6.5 \%$

$0.33[0.12,0.95] \quad 3.6 \%$

$0.13[0.06,0.24]$

$0.81[0.47,1.48] \quad 6.3 \%$

$0.39[0.19,0.82] \quad 5.3 \%$

$0.48[0.25,0.87]$

$1.85[0.88,3.90] \quad 5.3 \%$

$1.12[0.84,1.48] \quad 8.7 \%$

$0.88[0.46,1.71] \quad 5.8 \%$

$0.73[0.44,1.23] \quad 6.9 \%$

$0.51[0.10,2.28] \quad 2.2 \%$

$1.61[0.75,3.49] \quad 5.1 \%$

$0.84[0.62,1.12] \quad 8.6 \%$

$0.81[0.67,0.97] \quad 9.2 \%$

$1.1[0.8,1.5] \quad 8.5 \%$

$0.70[0.54,0.90] \quad 100 \%$

Heterogeneity: Tau $^{2}=0.17 ; \mathrm{Ch}^{2}=65.48, \mathrm{df}=15(p<0.00001) ; l^{2}=77 \%$

Test for overall effect: $Z=2.81 \quad(p=0.005)$

Myocardial infarction
Bozaite-Gluosniene et al. 2011
Burmester et al. 2012
Dixon et al. 2007
Greenberg et al. 2011
Jacobsson et al. 2005
Wolfe et al. 2008
All
Heterogeneity: Tau ${ }^{2}=0.22$; Chi
Test for overall effect: $Z=2.08$ (
Congestive heart failure
Bernatsky et al. 2005
Carmona et al. 2007
Jacobsson et al. 2005
Listing et al. 2008
Setoguchi et al. 2008
Solomon et al. 2012
Wolfe et al. 2004
All

Favors TNFi Favors no TNFi

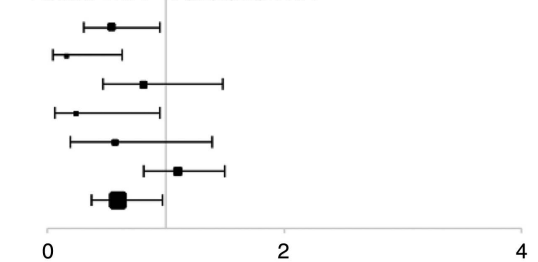

$0.54[0.30,0.95]$

$0.16[0.04,0.63]$

$0.81[0.47,1.48]$

$0.24[0.06,0.95]$

$0.57[0.20,1.39]$

$1.1[0.8,1.5]$

$0.59[0.36,0.97]$

$21.0 \%$

$8.9 \%$

$20.2 \%$

$8.9 \%$

$14.9 \%$

$26.1 \%$ $100 \%$

$0.5[0.2,0.9]$

$15.1 \%$

$0.14[0.04,0.37]-10.3 \%$

$0.00[0.00,1.13] \quad 0.4 \%$

$1.85[0.88,3.90] \quad 14.2 \%$

$1.61[0.75,3.49] \quad 13.7 \%$

$0.84[0.62,1.12] \quad 22.5 \%$

$0.81[0.67,0.97] \quad 23.9 \%$

$0.75[0.49,1.15] \quad 100 \%$

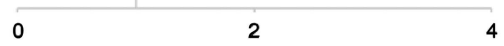

Heterogeneity: $\operatorname{Tau}^{2}=0.19 ; \mathrm{Ch}^{2}=25.66, d f=6(p=0.0003) ; R^{2}=77 \%$

Test for overall effect: $Z=1.31(p=0.19)$

Stroke
Burmester et al. 2012
Carmona et al. 2007
Greenberg et al. 2011
Jacobsson et al. 2005
Low et al. 2012
Nadareishvili et al. 2008
All

Favors TNFi Favors no TNFi

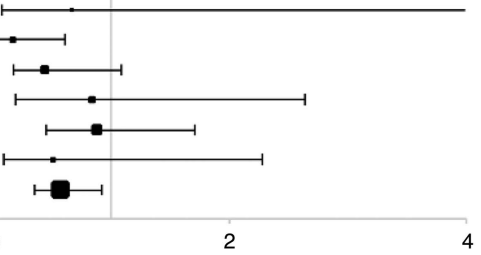

$0.67[0.09,5.20] \quad 5.2 \%$

$0.17[0.03,0.61] \quad 12.5 \%$

$0.44[0.18,1.09] \quad 22.3 \%$

$0.84[0.20,2.64] \quad 15.1 \%$

$0.88[0.46,1.71] \quad 35.5 \%$

$0.51[0.10,2.28] \quad 9.4 \%$

$0.57[0.35,0.92] \quad 100 \%$

Heterogeneity: $\operatorname{Tau}^{2}=0.05 ; \mathrm{Ch}^{2}=5.85, d f=5(p=0.32) ; R^{2}=15 \%$

Test for overall effect: $Z=2.29(p=0.02)$

MACE

Burmester et al. 2012

Carmona et al. 2007

Greenberg et al. 2011

Jacobsson et al. 2005

All

Heterogeneity: $\operatorname{Tau}^{2}=0.31 ; \mathrm{Ch}^{2}=10.02, d f=3(p=0.02) ;{ }^{2}=70 \%$

Test for overall effect: $Z=3.61$ ( $p=0.0003)$ $p=0.003$ ) (see online supplementary figure S3). No significant heterogeneity was observed (see online supplementary figure S4).

\section{DISCUSSION}

To our knowledge, this is the first systematic literature review and meta-analysis of published controlled studies assessing the 
association between CVEs and use of TNF inhibitors, methotrexate, NSAIDs or corticosteroids in patients with RA or Pso/PsA.

In patients with RA, treatment with TNF inhibitors or methotrexate was associated with a $30 \%$ and $28 \%$ reduction in the risk of CVEs, respectively, while use of NSAIDs or corticosteroids was associated with $18 \%$ and $47 \%$ increase in risk of all CVEs, respectively. Moreover, while TNF inhibitors and methotrexate were also found to be associated with a reduction in risk of some specific cardiovascular endpoints, corticosteroids were associated with an increase in risk of all specific outcomes. In patients with Pso/PsA, data suggest that biologics and other DMARDs may be associated with a decreased risk of CVEs, but we acknowledge that evidence is less conclusive than in RA and that further studies are needed.

Compared with the general population, patients with RA have an increased risk of cardiovascular disease or events ${ }^{50}$ and reduced survival. $^{2}$ Systemic inflammation is the cornerstone of both RA and atherosclerosis. However, whether effective DMARD-based therapy can ameliorate this increased risk in RA or Pso/PsA is still under debate. Considering these immunomodulatory strategies in atherosclerosis may also provide new perspective in managing cardiovascular disease in the general population. Indeed, in the (non-RA) primary cardiovascular disease prevention Justification for Use of statins in Prevention: an Intervention Trial Evaluating Rosuvastatin randomised controlled trial, the absolute risk of first major adverse cardiac events increased with increasing highsensitivity C-reactive protein (CRP) levels, a measure of systemic inflammation. $^{51} 52$ This study clearly showed an association between inflammation and cardiovascular risk in otherwise 'healthy' individuals. In RA, elevated baseline CRP has been associated with cardiovascular death, persisting after adjustment for classical cardiovascular risk factors, supporting a relevant link between systemic inflammation and risk of cardiovascular disease. ${ }^{5}$ Hence, by controlling the systemic inflammation, TNF inhibitors and methotrexate may decrease cardiovascular risk. Our present findings in RA support this hypothesis.

Several previous publications have also suggested that TNF inhibitors have a beneficial impact on cardiovascular risk. ${ }^{53} 54$ As suggested in a systematic literature review, ${ }^{55}$ another meta-analysis reported that TNF inhibitors in RA may reduce the risk of all CVEs (pooled adjusted RR, 0.46; 95\% CI 0.28 to 0.77 ), myocardial infarction (pooled adjusted RR, 0.81; 95\% CI 0.68 to 0.96 ) and cerebrovascular accident (pooled adjusted RR, 0.69 ; 95\% CI 0.53 to 0.89$).{ }^{15}$ Our analyses included more studies than those previously reported and our results are consistent with these prior findings. Interestingly, we found no association between TNF inhibitors and risk of heart failure in RA. In the general population with heart failure, TNF- $\alpha$ levels are increased $^{56}$ and associated with severity of clinical signs and symptoms. ${ }^{57}$ Experimental heart failure models have suggested that TNF inhibitors may improve ventricular dysfunction ${ }^{58}$; however, a large clinical trial assessing etanercept in the treatment of congestive heart failure showed no benefit ${ }^{59}$ while another one found that high-dose infliximab worsened heart failure in patients with moderate-to-severe chronic heart failure. ${ }^{60}$ Consequently, the presence of severe heart failure 
Figure 2 Continued

C Non-steroidal anti-inflammatory drugs (NSAIDs)

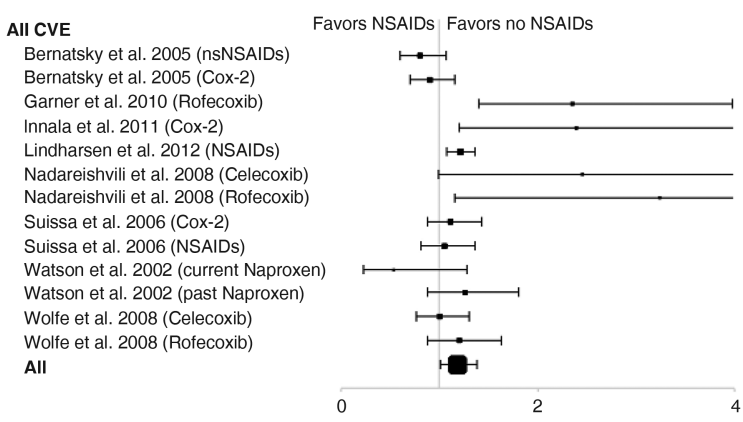

RR $[95 \% \mathrm{Cl}]$

$0.8[0.6,1.1] \quad 9.7 \%$

$0.9[0.7,1.2] \quad 10.5 \%$

$2.35[1.39,4.00] \quad 5.4 \%$

$2.39[1.21,4.74] \quad 3.9 \%$

$1.21[1.07,1.36] \quad 13.3 \%$

$2.45[0.99,5.45] \quad 3.0 \%$

$3.24[1.15,7.62] \quad 2.7 \%$

$1.11[0.87,1.43] \quad 10.7 \%$

$1.05[0.81,1.36] \quad 10.3 \%$

$0.53[0.22,1.28] \quad 2.6 \%$

$26[0.88,1.81] \quad 8.2 \%$

$\begin{array}{lr}1.0[0.8,1.3] & 10.3 \% \\ 1.2[0.9,1.6] & 9.3 \%\end{array}$

$1.18[1.01,1.38] \quad 100 \%$

Heterogeneity: $\operatorname{Tau}^{2}=0.04 ; C h{ }^{2}=35.64, d f=12(p=0.0004) ; P^{2}=66 \%$

Test for overall effect: $Z=2.08(p=0.04)$

All CVE - COX-2 inhibitors

Favors NSAIDs Favors no NSAIDs

Bernatsky et al. 2005 (Celecoxib or Rofecoxib)

Garner et al. 2010 (Rofecoxib)

Innala et al. 2011 (Cox-2)

Lindharsen et al. 2012 (Celecoxib)

Lindharsen et al. 2012 (Rofecoxib)

Nadareishvili et al. 2008 (Celecoxib)

Nadareishvili et al. 2008 (Rofecoxib)

Suissa et al. 2006 (Celecoxib or Rofecoxib)

Wolfe et al. 2008 (Celecoxib)

Wolfe et al. 2008 (Rofecoxib)

All

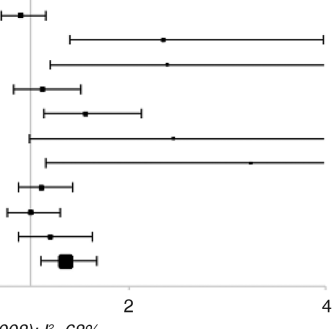

$0.9[0.7,1.2]$

$2.35[1.39,4.00]$

$2.39[1.21,4.74]$

$1.12[0.83,1.52]$

$1.56[1.14,2.13]$

$2.45[0.99,5.45]$

$3.24[1.15,7.62]$

$1.11[0.87,1.43]$

$1.0[0.8,1.3]$

$1.36[1.10,1.67]$

Heterogeneity: $\operatorname{Tau}^{2}=0.07 ; \mathrm{Ch} P=28.40, d f=9(p=0.0008) ; P^{2}=68 \%$

Test for overall effect: $Z=2.87(p=0.004)$

All CVE - Non-COX-2 NSAIDs Favors NSAIDs Favors no NSAIDs

Bernatsky ot al. 2005 (nSNSAIDS) Undharsen et al. 2012 (Naproxen) Undharsen et al. 2012 (Naproxen) Lindharsen et al. 2012 (Ketoprofen) Lindharsen et al. 2012 (Etodotac) Lindharsen et al. 2012 (Ibuprofen)
Lindharsen et al. 2012 (Diclofenac) Lindharsen et al. 2012 (Diclofenac) Watson et al. 2002 (current Naproxen) Watson et al. 2002 (past Naproxen)

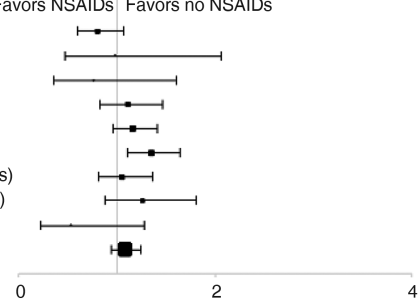

$0.8[0.6,1.1]$ $0.98[0.47,2.06]$ $0.76[0.36,1.60]$

$1.11[0.83,1.47$

$1.16[0.96,1.41]$

$1.35[1.11,1.64]$

$1.05[0.81,1.36]$

$1.26[0.88 .1 .81]$

$0.53[0.22 .1 .28]$

Al

$1.08[0.94,1.24]$

Heterogeneity: $\operatorname{Tau}^{2}=0.02 ; C h{ }^{2}=13.46, d f=8(p=0.10) ; P^{2}=41 \%$ Test for overall effect: $Z=1.08(p=0.28)$

Myocardial infarction

Favors NSAIDs Favors no NSAIDs Garner et al. 2010 (Rotecoxib) Suissa et al. 2006 (Cox-2) Suissa et al. 2006 (NSAIDs) Wolfe et al. 2008 (Celecoxib) Wolfe et al. 2008 (Rofecoxib) All

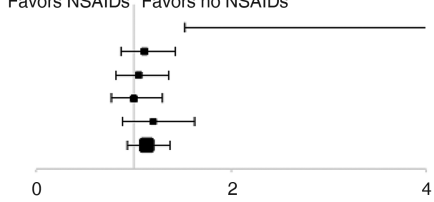

$4.48[1.52,13.13] \quad 3.0 \%$ $1.11[0.87,1.43]-25.7 \%$ $1.05[0.81,1.36] \quad 25.1 \%$ $1.0[0.8,1.3] \quad 24.9 \%$ $1.2[0.9,1.6] \quad 21.3 \%$ $1.13[0.93,1.37] \quad 100 \%$

Heterogeneity: $\operatorname{Tau}^{2}=0.02 ; \mathrm{Ch} P=7.49, \mathrm{df}=4(p=0.11) ; P^{2}=47 \%$ Test for overall effect: $Z=1.25(p=0.21)$

Congestive heart failure Bernatsky et al. 2005 (nsNSAIDs) Bernatsky et al. 2005 (Cox-2)

AlI

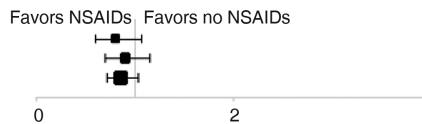

$0.8[0.6,1.1]$ $0.9[0.7,1.2]$ $0.86[0.71,1.03]$

Heterogeneity: $\operatorname{Tau}^{2}=0.00 ; C h \mathrm{P}^{2}=0.36, \mathrm{df}=1(p=0.55) ; \mathrm{R}^{2}=0 \%$ Test for overall effect: $Z=1.62(p=0.11)$

Stroke

Garner et al. 2010

Nadareishvili et al. 2008 (Celecoxib)

Nadareishvili et al. 2008 (Rofecoxib)

Favors NSAIDs Favors no NSAIDs

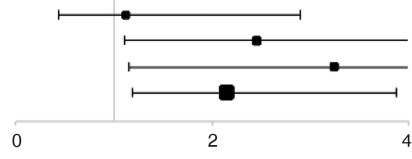

$12[0.43 .2 .90]$ $2.45[1.10,5.45]$ $3.24[1.15,7.62]$ $2.15[1.19,3.87]$

Heterogeneity: $\operatorname{Tau}^{2}=0.08 ; C h \boldsymbol{r}^{2}=2.79, d f=2(p=0.25) ; P^{2}=28 \%$ Test for overall effect: $Z=2.54(p=0.01)$

\section{MACE}

Innala et al. 2011

Lindharsen et al. 2012

All

Favors NSAIDs Favors no NSAIDs

- 0

Test for overall effect: $Z=1.35(p=0.18)$ 
Figure 2 Continued

D Corticosteroids

All CVE
Anandarajah et al. 2012
Avina-Zubieta et al. 2011
Avina-Zubieta et al. 2013
Davis et al. 2007 (GCS > 3 months)
Davis et al. 2007 (GCS $\leq 3$ months)
Greenberg et al. 2011 (Prednisone: $1-7 \mathrm{mg} / \mathrm{d}$ )
Greenberg et al. 2011 (Prednisone: $\geq 7 \mathrm{mg} / \mathrm{d}$ )
Innala et al. 2011
Nadareishvili et al. 2008
Solomon et al. 2012 ( Oral GCS: $1-4.9 \mathrm{mg}$ )
Solomon et al. 2012 (Oral GCS: $5+\mathrm{mg}$ )
Suissa et al. 2006
van Halm et al. 2006
Wolfe et al. 2008
All

0

Heterogeneity: $\operatorname{Tau}^{2}=0.00 ;$ Chi $^{2}=13.44, d f=12(p=0.34) ; P^{2}=11 \%$ Test for overall effect, $Z=7.60(p<0.00001)$

Myocardial infarction
Avina-Zubieta et al. 2013
Suissa et al. 2006
Wolfe et al. 2008
All

Favors steroids Favors no steroids

$1.68[1.14,2.47] \quad 14.0 \%$

$1.32[1.02,1.72] \quad 30.1 \%$

$1.4[1.0,1.7] \quad 55.9 \%$

$1.41[1.22,1.63] \quad 100 \%$

Heterogeneity: $\operatorname{Tau}^{2}=0.00 ; \mathrm{Ch}^{2}=1.03, d f=2(p=0.60) ; P^{2}=0 \%$

Test for overall effect, $Z=4.65(p<0.00001)$

Congestive heart failure

Congestive heart failure Favors steroids Favors no steroids
Solomon et al. 2012 (Oral GCS: $1-4.9 \mathrm{mg}$ )

Solomon et a 2012 (Oral GCS: $5+4.9 \mathrm{mg})$

All

$1.30[0.91,1.85] \quad 49.4 \%$

$1.54[1.09,2.19] \quad 50.6 \%$

$1.42[1.10,1.82] \quad 100 \%$

$$
0
$$

Heterogeneity: $\operatorname{Tau}^{2}=0.00 ; \mathrm{Ch}^{2}=0.44, d f=1 \quad(p=0.51) ; R^{R}=0 \%$

Test for overall effect, $Z=2.72(p=0.006)$

Stroke

Avina-Zubieta 2011

Nadareishvili et al. 2008

All

Favors steroids Favors no steroids

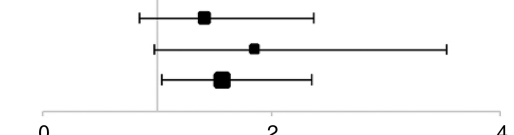

$1.41[0.84,2.37]$

$1.85[0.97,3.54]$

$61.0 \%$

$1.57[1.05,2.35] \quad 100 \%$

0

Heterogeneity: $\operatorname{Tau}^{2}=0.00 ; \mathrm{Ch}^{2}=0.44, d f=1 \quad(p=0.52) ; P^{2}=0 \%$

Test for overall effect, $Z=2.17(p<0.03)$

MACE

Anandarajah et al. 2012

Greenberg et al. 2011 (Prednisone: 1-7 mg/d)

Greenberg et al. 2011 (Prednisone: $\geq 7 \mathrm{mg} / \mathrm{d}$ )

Innala et al. 2011

van Halm et al. 2006

All
Favors steroids Favors no steroids

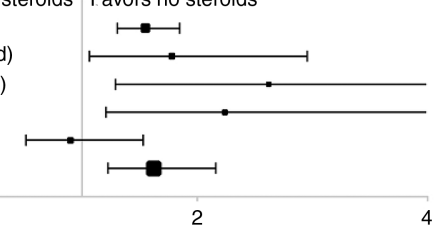

$1.55[1.30,1.85]$

$1.78[1.06,2.96]$

$2.62[1.29,5.31]$

$2.24[1.21,4.16]$

$0.90[0.51,1.53]$

$1.62[1.22,2.16]$
$37.6 \%$

$18.5 \%$

$11.9 \%$

$14.4 \%$

$17.6 \%$

$100 \%$

Heterogeneity: $\operatorname{Tau}^{2}=0.05 ; \mathrm{Chi}^{2}=7.75, d f=4(p=0.10) ;{ }^{2}=48 \%$

Test for overall effect, $Z=3.30(p=0.0010)$

remains a contraindication to the use of TNF inhibitors in patients with RA. This may account for our results, given that the selected studies probably included patients with RA without pre-existing heart failure, consistent with a bias by indication. However, one recent study comparing 8656 new users of nonbiological DMARDs with 11587 new users of TNF inhibitors showed that TNF inhibitors were not associated with an increased risk of hospital admissions due to heart failure. ${ }^{37}$ Further specifically designed trials assessing cardiovascular outcomes are needed to evaluate the use of TNF inhibitors in patients with RA, including those with heart failure.

In RA, methotrexate has recently been associated with a $70 \%$ reduction in mortality ${ }^{61}$ and may also be associated with a reduced risk of cardiovascular disease. ${ }^{62}$ Consistent with our results, methotrexate has been associated with a $21 \%$ lower risk of total cardiovascular disease and an 18\% lower risk of myocardial infarction in patients with chronic inflammatory diseases including RA. ${ }^{63}$ Corticosteroids and NSAIDs are generally considered to have deleterious effects, although little evidencebased data are available in patients with RA. Both non-selective NSAIDs and COX-2 inhibitors have been demonstrated to increase cardiovascular risk ${ }^{64-66}$; however, few studies have evaluated the impact of corticosteroids and NSAIDs on CVEs specifically in RA. ${ }^{17-22} 36{ }^{44}$ Our meta-analysis in patients with $\mathrm{RA}$ revealed that COX-2 inhibitors were associated with an increased risk of all CVEs and strokes, mainly because of rofecoxib, which is now withdrawn from the market. Conversely, non-selective NSAIDs and celecoxib were not associated with increased risk of CVEs. The practical consequence of this finding may be to weigh the benefit-risk ratio of using NSAIDs 

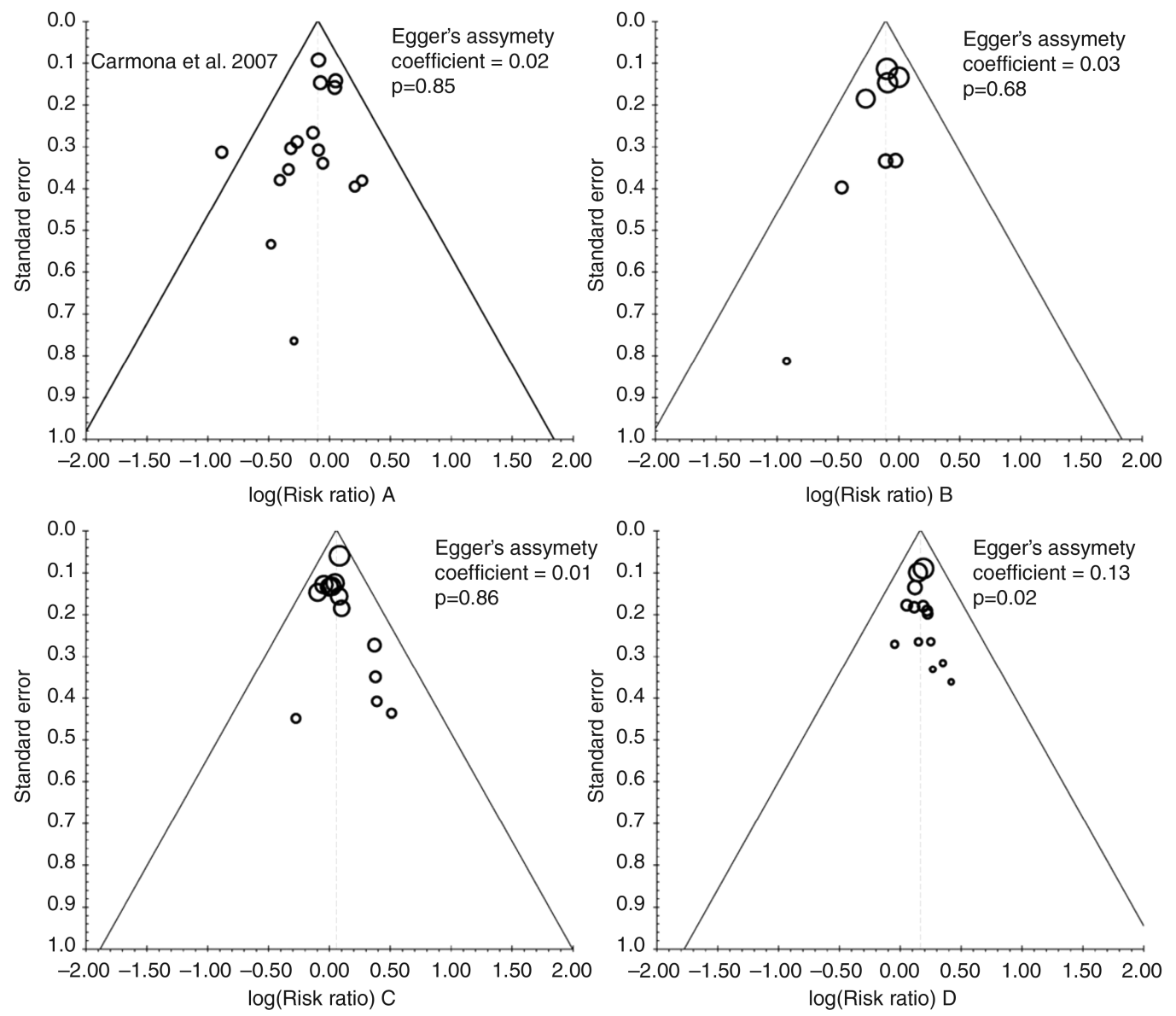

Figure 3 Funnel plots for the meta-analysis of occurrence of cardiovascular events associated with treatment with (A) tumour necrosis factor inhibitors, (B) methotrexate, (C) non-steroidal anti-inflammatory drugs and (D) corticosteroids. The importance (weight) of each study is proportional to the marker size.

in patients with RA with higher cardiovascular risk, while not avoiding them at all costs.

The potential harmful cardiovascular effects of corticosteroids are well-known but not strongly evidence based. ${ }^{65}$ To our knowledge, our meta-analysis is the first to report a deleterious association between corticosteroids and all CVEs, myocardial infarction, heart failure, stroke and major adverse cardiac events in the RA population. Corticosteroids may modulate the risk of cardiovascular disease in patients with RA in two competing directions: by increasing the risk due to deleterious effects on lipids, glucose tolerance, weight gain and hypertension, ${ }^{67}$ but conversely potentially decreasing the risk by exerting antiinflammatory and antiproliferative effects on vascular wall. Additionally, corticosteroids may result in two independent effects over time: an immediate effect of current exposure and a long-term cumulative effect of past exposure. ${ }^{22}{ }^{68}$ Notably, corticosteroid use has recently been associated with a dosedependent increased mortality in RA. ${ }^{69}$

In PsA/Pso, less evidence on risk of CVEs has been published. However, the results of our meta-analysis are consistent with recent reviews, ${ }^{70}{ }^{71}$ underlining the need for adequately powered trials to assess the cardiovascular effects of systemic therapy including TNF inhibitors in patients with PsA/Pso.

Interpreting the evidence from observational studies requires caution as these can generate more bias than randomised controlled trials. Hence, only an association between medication use and CVEs can be determined. However, we were unable to find any adequately powered randomised controlled trial assessing the impact of RA therapy on risk of CVEs. Furthermore, the duration of follow-up of most randomised controlled trials in patients with RA or PsA/Pso may not be long enough to investigate the effects of such treatments on CVE rates. Finally, our results are consistent with two large meta-analyses evaluating methotrexate and TNF inhibitors in RA. ${ }^{15} 63$

Despite the mounting published evidence of an increased cardiovascular risk in patients with RA, few studies have investigated DMARD-based therapeutic strategies to reduce this risk. Therefore, a low number of studies assessing the impact of methotrexate on specific cardiovascular endpoints were included in our analysis. Additionally, lack of sufficient data did not allow for subgroup analyses of dose effect for methotrexate and corticosteroids in RA, or of the influence of psoriasis severity on the impact of systemic therapy on CVE rates in patients with PsA/ Pso. No study evaluating the impact of non-TNF inhibitor biologics was found, although several studies evaluating the vascular impact of rituximab (NCT00844714) and tocilizumab (NCT00844714 and NCT01752335) are ongoing in RA.

Another limitation in this analysis is the differences in the cardiovascular definitions and comparators across studies. To mitigate this, we used selection criteria that would provide as homogeneous as possible study populations to provide clinically relevant results.

\section{CONCLUSION}

Our meta-analysis provides important insights into the effect of antirheumatic drugs on CVEs in RA by suggesting that TNF inhibitors and methotrexate decrease the risk of such events while NSAIDs and corticosteroids increase the risk in RA. In 
PsA/Pso, limited evidence suggests that systemic therapies are associated with a decrease in the risk of all CVEs. While current evidence implies deleterious cardiovascular effects of corticosteroids and COX-2 inhibitors in RA, suppressing diverse mediators of inflammation with methotrexate and TNF inhibitors may have positive cardiovascular effects. Large, prospective, adequately controlled and powered studies are needed to explore the effects of such drugs on cardiovascular morbidity and mortality in the RA and PsA/Pso populations.

\section{Author affiliations} \\ ${ }^{1}$ University of Montreal Hospital Research Center (CRCHUM), Notre-Dame Hospital, \\ Montreal, Quebec, Canada \\ ${ }^{2}$ Department of Medicine, Dermatology Service, St-Luc Hospital, Montreal, Quebec, \\ Canada \\ ${ }^{3}$ Sacré-Coeur Hospital of Montreal, University of Montreal, Montreal, Quebec, \\ Canada \\ ${ }^{4}$ Department of Dermatology and Skin Science, University of British Columbia, \\ Vancouver, British Columbia, Canada \\ ${ }^{5}$ Division of Rheumatology, University of Alberta, Edmonton, Alberta, Canada \\ ${ }^{6}$ Division of Dermatology, University of Toronto, Toronto, Ontario, Canada \\ ${ }^{7}$ Division of Rheumatology, Department of Medicine, Western University of Canada, \\ St. Joseph's Health Care, London, Ontario, Canada \\ ${ }^{8}$ Lynde Dermatology, Markham, Ontario, Canada \\ ${ }^{9}$ Faculty of Medicine, Memorial University of Newfoundland, St. John's, \\ Newfoundland, Canada \\ ${ }^{10}$ Department of Medicine, Centre de Recherche du CHU de Québec, Laval \\ University, Quebec City, Quebec, Canada \\ ${ }^{11}$ Innovaderm Research, Montreal, Quebec, Canada \\ ${ }^{12}$ Department of Medicine, Rheumatic Disease Unit, Centre Hospitalier de \\ I'Université de Montréal (CHUM) and Institut de Rhumatologie de Montréal, \\ Montreal, Quebec, Canada
}

Acknowledgements The authors would like to acknowledge Dr Fred Wolfe and Dr Lotta Ljung, who kindly answered the questions of the authors, Mr Andre Allard (librarian, Montreal, QC, Canada), who helped perform the systemic literature search, and Mr Louis Coupal (Saint-Lambert, QC, Canada), who helped perform the statistical analysis. CR received a bursary from the Foundation of the University of Montreal Hospital Center (CHUM) for her fellowship. CM was the recipient of a Janssen Ortho Canada fellowship and a Geoffrey Dowling fellowship from the British Association of Dermatologists.

Contributors All authors are responsible for the work described in this paper. All authors were involved in at least one of the following: conception, design, acquisition, analysis, statistical analysis and interpretation of data. All authors were involved in drafting the manuscript and/or reviewing the manuscript for important intellectual content. All authors provided final approval of the version to be published.

Funding This publication summarises the results of the Canadian Dermatology-Rheumatology (DR) Co-morbidity Initiative systematic literature searches (MEDLINE, EMBASE, Cochrane Library, 2010-2012 ACR, EULAR, AAD, EADV abstracts) and consensus-based recommendations from a meeting on the management of comorbidities, held in Toronto, Canada, on 31 May to 1 June 2013, which was sponsored by AbbVie. AbbVie provided funding to Pinnacle Marketing \& Education Inc., 7 Daoust, Hudson, Quebec, Canada, to manage the Canadian DR Co-morbidity Initiative that led to this paper. AbbVie paid consultancy fees to $\mathrm{BH}$, $J P, L B, S K, R B, W G, J D, C L$ and JK for their participation in the Canadian DR Co-morbidity Initiative. Travel to and from the meeting was reimbursed. AbbVie provided suggestions for topic ideas for the Canadian DR Co-morbidity Initiative and proposed authors for this paper. AbbVie attended the meeting but was not involved in the development of the paper. AbbVie did have the opportunity to review the final version of the publication. This publication reflects the opinions of the authors. CR prepared a draft outline for coauthors' comment and approval. Each author reviewed the publication at all stages of development to ensure that it accurately reflects the results of their literature searches and the consensus-based recommendations from the meeting experts. The authors determined final content, and all authors read and approved the final publication. The authors maintained complete control over the content of the paper. No payments were made to the authors for the writing of this publication. Juliette Allport of Leading Edge (part of the Lucid Group), Burleighfield House, Buckinghamshire, UK, subsequently supported incorporation of comments into final drafts for author approval, and editorial styling required by the journal. AbbVie paid Leading Edge for this work.

Competing interests AM owns stock in Pfizer. JK has acted as consultant, advisor and/or speaker for AbbVie, Amgen, Janssen, Leo Pharma and Novartis. CL has acted as a principal investigator, speaker and/or consultant for AbbVie, Amgen, Celgene, Eli Lilly, Galderma, Janssen, Leo Pharma, Merck, Novartis, Pfizer and
Valeant. JP has received research grants and/or has been a consultant for AbbVie, Actelion, Amgen, Bristol-Myers Squibb, Celgene, Janssen, Pfizer, Roche and UCB. SK has received unrestricted educational grants and consultancy fees from AbbVie, Amgen, AstraZeneca, Janssen, Roche and UCB. JD has received honoraria for participation in advisory boards and as a speaker for AbbVie, Amgen, Janssen and Leo Pharma. LB has received consulting fees, research grants, and honoraria for participation as a speaker for AbbVie, Amgen, Bristol-Myers Squibb, Janssen, Pfizer, Roche and UCB. RB has received honoraria for participation in advisory boards and/ or research grants from AbbVie, Amgen, Celgene, Eli-Lilly Galderma, Incyte, Janssen, Leo Pharma, Merck, Novartis, Pfizer and Tribute. BH has received honoraria for participation in advisory boards and/or research grants from AbbVie, Amgen, Bristol-Myers Squibb, Janssen, Pfizer, Roche and UCB. WG has received honoraria for participation in advisory boards for AbbVie, Amgen, Bio-K and Janssen and for participation in speaker engagements and consultative meetings for AbbVie, Actelion, Amgen, Janssen, Leo Pharma, Novartis and Roche.

Provenance and peer review Not commissioned; externally peer reviewed.

Open Access This is an Open Access article distributed in accordance with the Creative Commons Attribution Non Commercial (CC BY-NC 4.0) license, which permits others to distribute, remix, adapt, build upon this work non-commercially, and license their derivative works on different terms, provided the original work is properly cited and the use is non-commercial. See: http://creativecommons.org/ licenses/by-nc/4.0/

\section{REFERENCES}

1 Nicola PJ, Maradit-Kremers $H$, Roger VL, et al. The risk of congestive heart failure in rheumatoid arthritis: a population-based study over 46 years. Arthritis Rheum 2005;52:412-20.

2 Solomon DH, Karlson EW, Rimm EB, et al. Cardiovascular morbidity and mortality in women diagnosed with rheumatoid arthritis. Circulation 2003;107:1303-7.

3 Coumbe AG, Pritzker MR, Duprez DA. Cardiovascular risk and psoriasis: beyond the traditional risk factors. Am J Med 2014;127:12-18.

4 Dowlatshahi EA, Kavousi M, Nijsten T, et al. Psoriasis is not associated with atherosclerosis and incident cardiovascular events: the Rotterdam Study. J Invest Dermatol 2013;133:2347-54.

5 Goodson NJ, Symmons DP, Scott DG, et al. Baseline levels of C-reactive protein and prediction of death from cardiovascular disease in patients with inflammatory polyarthritis: a ten-year followup study of a primary care-based inception cohort. Arthritis Rheum 2005;52:2293-9.

6 Kerola AM, Kerola T, Kauppi MJ, et al. Cardiovascular comorbidities antedating the diagnosis of rheumatoid arthritis. Ann Rheum Dis 2013;72:1826-9.

7 Roubille $F$, Kritikou EA, Roubille $C$, et al. Emerging anti-inflammatory therapies for atherosclerosis. Curr Pharm Des 2013;19:5840-9.

8 Roubille C, Haraoui B. Important issues at heart: cardiovascular risk management in rheumatoid arthritis. Ther Adv Musculoskelet Dis 2013;5:163-5.

9 Smolen JS, Landewe R, Breedveld FC, et al. EULAR recommendations for the management of rheumatoid arthritis with synthetic and biological disease-modifying antirheumatic drugs. Ann Rheum Dis 2010;69:964-75.

10 Peters MJ, Symmons DP, McCarey D, et al. EULAR evidence-based recommendations for cardiovascular risk management in patients with rheumatoid arthritis and other forms of inflammatory arthritis. Ann Rheum Dis 2010;69:325-31.

11 Moher D, Liberati A, Tetzlaff J, et al. Preferred reporting items for systematic reviews and meta-analyses: the PRISMA statement. Ann Intern Med 2009;151:264-9, W64.

12 Prodanovich S, Ma F, Taylor JR, et al. Methotrexate reduces incidence of vascular diseases in veterans with psoriasis or rheumatoid arthritis. J Am Acad Dermatol 2005;52:262-7.

13 Al-Aly Z, Pan H, Zeringue A, et al. Tumor necrosis factor-alpha blockade, cardiovascular outcomes, and survival in rheumatoid arthritis. Trans/ Res 2011;157:10-18.

14 Abou-Setta AM, Beaupre LA, Rashiq $S$, et al. Comparative effectiveness of pain management interventions for hip fracture: a systematic review. Ann Intern Med 2011;155:234-45.

15 Barnabe C, Martin BJ, Ghali WA. Systematic review and meta-analysis: anti-tumor necrosis factor alpha therapy and cardiovascular events in rheumatoid arthritis. Arthritis Care Res (Hoboken) 2011;63:522-9.

16 Zhang J, Yu KF. What's the relative risk? A method of correcting the odds ratio in cohort studies of common outcomes. JAMA 1998;280:1690-1.

17 Nadareishvili Z, Michaud K, Hallenbeck JM, et al. Cardiovascular, rheumatologic, and pharmacologic predictors of stroke in patients with rheumatoid arthritis: a nested, case-control study. Arthritis Rheum 2008;59:1090-6.

18 Watson DJ, Rhodes T, Cai B, et al. Lower risk of thromboembolic cardiovascular events with naproxen among patients with rheumatoid arthritis. Arch Intern Med 2002;162:1105-10.

19 Suissa S, Bernatsky S, Hudson M. Antirheumatic drug use and the risk of acute myocardial infarction. Arthritis Rheum 2006;55:531-6. 
20 Davis JM III, Maradit Kremers H, Crowson CS, et al. Glucocorticoids and cardiovascular events in rheumatoid arthritis: a population-based cohort study. Arthritis Rheum 2007;56:820-30.

21 Avina-Zubieta JA, Abrahamowicz M, Choi HK, et al. Risk of cerebrovascular disease associated with the use of glucocorticoids in patients with incident rheumatoid arthritis: a population-based study. Ann Rheum Dis 2011;70:990-5.

22 Avina-Zubieta JA, Abrahamowicz M, De Vera MA, et al. Immediate and past cumulative effects of oral glucocorticoids on the risk of acute myocardial infarction in rheumatoid arthritis: a population-based study. Rheumatology (Oxford) 2013;52:68-75.

23 Bernatsky S, Hudson M, Suissa S. Anti-rheumatic drug use and risk of hospitalization for congestive heart failure in rheumatoid arthritis. Rheumatology (Oxford) 2005:44:677-80.

24 Carmona L, Descalzo MA, Perez-Pampin E, et al. All-cause and cause-specific mortality in rheumatoid arthritis are not greater than expected when treated with tumour necrosis factor antagonists. Ann Rheum Dis 2007;66:880-5.

25 Dixon WG, Watson KD, Lunt $M$, et al. Reduction in the incidence of myocardial infarction in patients with rheumatoid arthritis who respond to anti-tumor necrosis factor alpha therapy: results from the British Society for Rheumatology Biologics Register. Arthritis Rheum 2007;56:2905-12.

26 Choi HK, Hernan MA, Seeger JD, et al. Methotrexate and mortality in patients with rheumatoid arthritis: a prospective study. Lancet 2002;359:1173-7.

27 Garner SE, Fidan DD, Frankish RR, et al. Rofecoxib for rheumatoid arthritis. Cochrane Database Syst Rev 2005:CD003685.

28 Greenberg JD, Kremer JM, Curtis JR, et al. Tumour necrosis factor antagonist use and associated risk reduction of cardiovascular events among patients with rheumatoid arthritis. Ann Rheum Dis 2011;70:576-82.

29 Innala L, Moller B, Ljung L, et al. Cardiovascular events in early RA are a result of inflammatory burden and traditional risk factors: a five year prospective study. Arthritis Res Ther 2011;13:R131.

30 Jacobsson LT, Turesson C, Gulfe A, et al. Treatment with tumor necrosis factor blockers is associated with a lower incidence of first cardiovascular events in patients with rheumatoid arthritis. J Rheumatol 2005;32:1213-18.

31 Listing J, Strangfeld A, Kekow J, et al. Does tumor necrosis factor alpha inhibition promote or prevent heart failure in patients with rheumatoid arthritis? Arthritis Rheum 2008;58:667-77.

32 Lunt $M$, Watson KD, Dixon WG, et al. No evidence of association between anti-tumor necrosis factor treatment and mortality in patients with rheumatoid arthritis: results from the British Society for Rheumatology Biologics Register. Arthritis Rheum 2010;62:3145-53.

33 Setoguchi S, Schneeweiss S, Avorn J, et al. Tumor necrosis factor-alpha antagonist use and heart failure in elderly patients with rheumatoid arthritis. Am Heart J 2008;156:336-41.

34 van Halm VP, Nurmohamed MT, Twisk JW, et al. Disease-modifying antirheumatic drugs are associated with a reduced risk for cardiovascular disease in patients with rheumatoid arthritis: a case control study. Arthritis Res Ther 2006;8:R151.

35 Wolfe F, Michaud K. Heart failure in rheumatoid arthritis: rates, predictors, and the effect of anti-tumor necrosis factor therapy. Am J Med 2004;116:305-11.

36 Wolfe F, Michaud K. The risk of myocardial infarction and pharmacologic and nonpharmacologic myocardial infarction predictors in rheumatoid arthritis: a cohort and nested case-control analysis. Arthritis Rheum 2008;58:2612-21.

37 Solomon $\mathrm{DH}$, Rassen JA, Kuriya $\mathrm{B}$, et al. Heart failure risk among patients with rheumatoid arthritis starting a TNF antagonist. Ann Rheum Dis 2013;72:1813-18.

38 Low AS, Lunt M, Mercer LK, et al. Association between anti-tumor necrosis factor therapy and the risk of ischemic stroke in subjects with rheumatoid arthritis. Results from the British Society For Rheumatology Biologics Registers-Rheumatoid Arthritis (BSRBR-RA). [abstract]. Arthritis Rheum 2012;64:828.

39 Anandarajah AP, Saunders KC, Reed GW, et al. Major adverse cardiovascular events are more common in rheumatoid arthritis than in psoriatic arthritis and are associated with different risk factors. [abstract]. Arthritis Rheum 2012;64:2221.

40 Bozaite-Gluosniene R, Tang X, Kirchner HL, et al. Reduced cardiovascular risk with use of methotrexate and tumor necrosis factor-inhibitors in patients with rheumatoid arthritis. [abstract]. Arthritis Rheum 2011;63:719.

41 Biki A, Tang $\mathrm{X}$, Kirchner $\mathrm{HL}$, et al. Prolonged hydroxychloroquine use is associated with decreased incidence of cardiovascular disease in rheumatoid arthritis patients. [abstract]. Arthritis Rheum 2011;63:1168.

42 Burmester G, Emery P, Signorovitch J, et al. The effects of adalimumab on risk of major adverse cardiovascular events in rheumatoid arthritus: a meta-analysis of randomized trials. [abstract]. Ann Rheum Dis 2012;71:359.

43 Ljung L, Rantapää-Dahlqvist S, Askling J, et al. The risk of acute coronary syndromes in RA in relation to TNF inhibitors and risks in the general populatiohn: a national cohort study. [abstract]. Ann Rheum Dis 2012;71:518.

44 Lindhardsen J, Gislason GH, Jacobsen S, et al. Non-steroidal anti-inflammatory drugs and risk of cardiovascular disease in patients with rheumatoid arthritis: a nationwide cohort study. Ann Rheum Dis 2014;73:1515-21.

45 Abuabara K, Lee $H$, Kimball AB. The effect of systemic psoriasis therapies on the incidence of myocardial infarction: a cohort study. $\mathrm{Br} J$ Dermatol 2011;165:1066-73.
46 Ahlehoff O, Skov L, Gislason G, et al. Cardiovascular disease event rates in patients with severe psoriasis treated with systemic anti-inflammatory drugs: a Danish real-world cohort study. J Intern Med 2013;273:197-204.

47 Chen YJ, Chang YT, Shen JL, et al. Association between systemic antipsoriatic drugs and cardiovascular risk in patients with psoriasis with or without psoriatic arthritis: a nationwide cohort study. Arthritis Rheum 2012;64:1879-87.

48 Lan CC, Ko YC, Yu HS, et al. Methotrexate reduces the occurrence of cerebrovascular events among Taiwanese psoriatic patients: a nationwide population-based study. Acta Derm Venereol 2012;92:349-52.

49 Wu JJ, Poon KY, Channual JC, et al. Association between tumor necrosis factor inhibitor therapy and myocardial infarction risk in patients with psoriasis. Arch Dermatol 2012;148:1244-50.

50 Avina-Zubieta JA, Thomas J, Sadatsafavi M, et al. Risk of incident cardiovascular events in patients with rheumatoid arthritis: a meta-analysis of observational studies. Ann Rheum Dis 2012;71:1524-9.

51 Ridker PM, Danielson E, Fonseca FA, et al. Rosuvastatin to prevent vascular events in men and women with elevated C-reactive protein. $N$ Engl J Med 2008;359:2195-207

52 Ridker PM, MacFadyen J, Libby P, et al. Relation of baseline high-sensitivity C-reactive protein level to cardiovascular outcomes with rosuvastatin in the Justification for Use of statins in Prevention: an Intervention Trial Evaluating Rosuvastatin (JUPITER). Am J Cardiol 2010;106:204-9.

53 Maki-Petaja KM, Elkhawad M, Cheriyan J, et al. Anti-tumor necrosis factor-alpha therapy reduces aortic inflammation and stiffness in patients with rheumatoid arthritis. Circulation 2012;126:2473-80.

54 Solomon DH, Curtis JR, Saag KG, et al. Cardiovascular risk in rheumatoid arthritis: comparing TNF-alpha blockade with nonbiologic DMARDs. Am J Med 2013;126:730.e9-e17

55 Westlake SL, Colebatch AN, Baird J, et al. Tumour necrosis factor antagonists and the risk of cardiovascular disease in patients with rheumatoid arthritis: a systematic literature review. Rheumatology (Oxford) 2011;50:518-31.

56 Levine B, Kalman J, Mayer $\mathrm{L}$, et al. Elevated circulating levels of tumor necrosis factor in severe chronic heart failure. N Engl J Med 1990;323:236-41.

57 McMurray J, Abdullah I, Dargie HJ, et al. Increased concentrations of tumour necrosis factor in "cachectic" patients with severe chronic heart failure. $\mathrm{Br}$ Heart $\mathrm{J}$ 1991;66:356-8

58 Kadokami T, Frye C, Lemster B, et al. Anti-tumor necrosis factor-alpha antibody limits heart failure in a transgenic model. Circulation 2001;104:1094-7.

59 Mann DL, McMurray JJ, Packer M, et al. Targeted anticytokine therapy in patients with chronic heart failure: results of the Randomized Etanercept Worldwide Evaluation (RENEWAL). Circulation 2004;109:1594-602.

60 Chung ES, Packer M, Lo KH, et al. Randomized, double-blind, placebo-controlled, pilot trial of infliximab, a chimeric monoclonal antibody to tumor necrosis factor-alpha, in patients with moderate-to-severe heart failure: results of the anti-TNF Therapy Against Congestive Heart Failure (ATTACH) trial. Circulation 2003;107:3133-40.

61 Wasko MC, Dasgupta A, Hubert $\mathrm{H}$, et al. Propensity-adjusted association of methotrexate with overall survival in rheumatoid arthritis. Arthritis Rheum 2013:65:334-42

62 Westlake SL, Colebatch AN, Baird J, et al. The effect of methotrexate on cardiovascular disease in patients with rheumatoid arthritis: a systematic literature review. Rheumatology (Oxford) 2010;49:295-307.

63 Micha R, Imamura F, Wyler von Ballmoos M, et al. Systematic review and meta-analysis of methotrexate use and risk of cardiovascular disease. Am J Cardiol 2011;108:1362-70.

64 Trelle S, Reichenbach S, Wandel S, et al. Cardiovascular safety of non-steroidal anti-inflammatory drugs: network meta-analysis. BMJ 2011;342:c7086.

65 Roubille C, Martel-Pelletier J, Davy JM, et al. Cardiovascular adverse effects of anti-inflammatory drugs. Antiinflamm Antiallergy Agents Med Chem 2013;12:55-67.

66 Coxib and traditional NSAID Trialists' (CNT) CollaborationBhala N, Emberson J, Merhi A, et al. Vascular and upper gastrointestinal effects of non-steroidal anti-inflammatory drugs: meta-analyses of individual participant data from randomised trials. Lancet 2013;382:769-79.

67 Panoulas VF, Douglas KM, Stavropoulos-Kalinoglou A, et al. Long-term exposure to medium-dose glucocorticoid therapy associates with hypertension in patients with rheumatoid arthritis. Rheumatology (Oxford) 2008;47:72-5.

68 Wei L, MacDonald TM, Walker BR. Taking glucocorticoids by prescription is associated with subsequent cardiovascular disease. Ann Intern Med 2004;141:764-70.

69 del Rincon I, Battafarano DF, Restrepo JF, et al. Glucocorticoid dose thresholds associated with all-cause and cardiovascular mortality in rheumatoid arthritis. Arthritis Rheumatol 2014;66:264-72.

70 Hugh J, Van Voorhees AS, Nijhawan RI, et al. From the Medical Board of the National Psoriasis Foundation: The risk of cardiovascular disease in individuals with psoriasis and the potential impact of current therapies. J Am Acad Dermatol 2014;70:168-77.

71 Armstrong AW, Brezinski EA, Follansbee MR, et al. Effects of biologic agents and other Disease-Modifying Antirheumatic Drugs on cardiovascular outcomes in psoriasis and psoriatic arthritis: a systematic review. Curr Pharm Des 2014;20:500-12. 